\title{
The Correlation Between Improvements In Telecommunications Technology And Growth Of Cities
}

Alexandros Panayides, William Paterson University, USA

\begin{abstract}
This paper examines the correlation between improvements in telecommunication technology and growth of cities. By developing a general and then an urban model we want to examine the impact of telecommunication improvements on city growth assuming first that face-to-face interactions and electronic communications are substitutes and then they are complements. The placement of the general model within an urban framework shows that city size increases with electronic communications if face-to-face interactions and electronic communications are complements.
\end{abstract}

Keywords: please use the following: Information Technology and Growth of Cities

\section{INTRODUCTION}

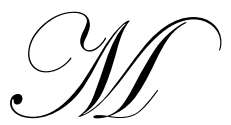

any futurists have argued that as telecommunications technology improves, the demand for faceto-face interactions, and thus will make cities, in which face-to-face interactions are facilitated, obsolete (Toffler (1980), Negroponte (1995), Naisbitt (1995), and Knoke (1996). Gaspar and Glaeser (1998) developed a model, to examine this relationship between telecommunications improvements and growth of cities, and showed that advanced communications technology can either increase or decrease both face-toface interactions and city size. In their model Gaspar and Glaeser (1998) assume that only one mode of interaction, either telecommunications or face-to-face interactions, can be used in a relationship. Panayides (2002) developed a model where individuals involved in a relationship use two modes of interaction: face-to-face interactions and electronic communications. Each individual maximizes her net payoff from the relationship. Then using comparative static analysis on the optimal solutions showed that improvements in telecommunications technology may increase or decrease the demand for face-to-face interactions, depending whether face-to-face interactions and telecommunications are substitutes or complements. Then this general model was placed within a spatial framework. The model presented here expands the Panayides (2002) and Gaspar and Glaeser (1998) models.

\section{THE PAYOFF MODEL}

In this section we construct a general payoff model using a quadratic function such that first face-to-face interactions and electronic communications are substitutes and then such that face-to-face interactions and electronic communications are complements.

General Payoff Model I (Face-to-face interactions and electronic communications are substitutes): An agent involved in relationships with other agents uses electronic communications and face-to-face contact. The payoff of the relationships depends on the time spent on electronic and face-to-face interactions and is given by the following function,

$$
V=a_{e} t_{e}+a_{f} b t f_{f}-\frac{1}{2} a_{e e} t_{e}^{2}-\frac{1}{2} a_{f f} b^{2} t_{f}^{2}-a_{e f} t_{e}^{b t} f
$$


where: $t_{e}$ is the time spent on electronic communications, $t_{f}$ is the time spent on face-to-face communications, $b$ is the fraction of face-to-face communication that is productive, $a_{e}$ is the parameter that increases the productivity of electronic communications, and $a_{f}$ is the parameter that increases the value of face-to-face interactions.

Note first, that $\frac{\partial^{2} V}{\partial t_{e} \partial b t_{f}}=-a_{e f}<0$, implying that face-to-face interactions and electronic communications are substitutes, second $\frac{\partial^{2} V}{\partial t_{e} \partial a_{f}}=0$, implying that more valuable face-to-face interactions do not increase the payoff from electronic communications, and third, $\frac{\partial^{2} V}{\partial b t_{f} \partial a_{e}}=0$, implying that better electronic communications do not make face-to-face interactions more valuable.

Time is a common input for both modes of interaction, which is available at cost $\mathrm{c}$ per unit. The total cost of engaging in interactions is then given by the following function,

$$
C=c\left(t_{e}+t_{f}\right)
$$

The objective of the agent is individual is to maximize her net payoff from interactions,

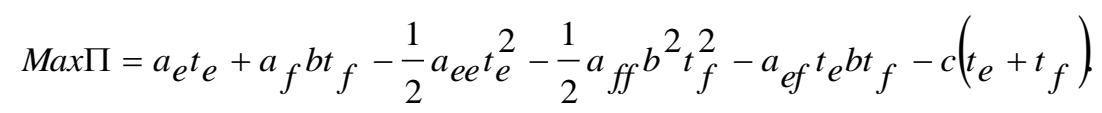

The first order conditions (FOCs) for payoff maximization are

$$
\begin{aligned}
& \frac{\partial \Pi}{\partial t_{e}}=a_{e}-a_{e e} t_{e}-a_{e f} b t_{f}-c=0, \\
& \frac{\partial \Pi}{\partial t_{f}}=b a_{f}-a_{f f} b^{2} t_{f}-a_{e f} b t_{e}-c=0 .
\end{aligned}
$$

Both first order conditions imply that the marginal payoff from either mode of interaction must be equal with the marginal cost which is the opportunity cost of time.

The second order conditions (SOCs) for a maximum are that the following Hessian determinant be positive:

$$
|H|=\left|\begin{array}{cc}
-a_{e e} & -b a_{e f} \\
-b a_{e f} & -b^{2} a_{f f}
\end{array}\right|=b^{2}\left(a_{e e} a_{f f}-a_{e f}^{2}\right)>0
$$

Using (3) and (4) the following optimal solutions for $t_{e}^{*}$ and $t_{f}^{*}$ are obtained:

$$
t_{f}^{*}=\frac{b a_{e e} a_{f}-b a_{e f} a_{e}+b c a_{e f}-c a_{e e}}{|H|},
$$


$t_{e}^{*}=\frac{a_{e}-c}{a_{e e}}-\frac{b a_{e f}}{a_{e e}} t_{f}^{*}$

We now investigate how an improvement in telecommunications technology affects the time spent on either mode of interaction. These effects are,

$$
\begin{aligned}
& \frac{\partial t_{e}^{*}}{\partial a_{e}}=\frac{1}{a_{e e}}-\left(\frac{b a_{e f}}{a_{e e}}\right)\left(\frac{\partial t_{f}^{*}}{\partial a_{e}}\right)>0, \\
& \frac{\partial t_{f}^{*}}{\partial a_{e}}=\frac{-b a_{e f}}{|H|}<0 .
\end{aligned}
$$

In addition the following comparative static result is needed to determine the effect of advances in information technology on city size,

$$
\frac{\partial t_{e}^{*}}{\partial b}=-\frac{c a_{e f}}{|H|}<0
$$

Result (8) implies that improvements in communications technology will increase the time spent on electronic communications since now it's easier and faster to communicate electronically. Therefore, if face-to-face interactions and electronic communications are substitutes, improvements in communications technology will decrease the time spent on face-to-face interactions as people substitute face-to-face contact with electronic communications, which is what result (9) implies. Finally, result (10) implies that an increase in the productive time of face-to-face contact will decrease the time spent on electronic contact, as people substitute electronic with faceto-face contact.

General Payoff Model II: (Face-to-face interactions and electronic communications are complements): The payoff from the relationships is now given by the following function,

$V=a_{e} e_{e}+a_{f} b t f-\frac{1}{2} a_{e e} t_{e}^{2}-\frac{1}{2} a_{f f} b^{2} t_{f}^{2}+a_{e f} t_{e}^{b t} f$

Note that $\frac{\partial^{2} V}{\partial t_{e} \partial b t_{f}}=a_{e f}>0$, implying that face-to-face interactions and electronic communications are complements. As in model I, the individual maximizes her net payoff form the relationships. Namely, she is faced with the following problem:

$$
\operatorname{Max} \Pi=a_{e} t_{e}+a_{f} b t_{f}-\frac{1}{2} a_{e e} t_{e}^{2}-\frac{1}{2} a f f b^{2} t_{f}^{2}+a_{e f} t_{e}^{b t} f-c\left(t_{e}+t_{f}\right)
$$

Following similar analysis as in the general model I, the following optimal solutions can be obtained: 


$$
\begin{aligned}
& t_{f}^{*}=\frac{b a_{e e} a_{f}+b a_{e f} a_{e}-b c a_{e f}-c a_{e e}}{|H|}, \\
& t_{e}^{*}=\frac{a_{e}-c}{a_{e e}}+\frac{b a_{e f}}{a_{e e}} t_{f}^{*} .
\end{aligned}
$$

We now investigate how an improvement in telecommunications technology affects the time spent on either mode of interaction. These effects are,

$$
\begin{aligned}
& \frac{\partial t_{e}^{*}}{\partial a_{e}}=\frac{1}{a_{e e}}+\left(\frac{b a_{e f}}{a_{e e}}\right)\left(\frac{\partial t_{f}^{*}}{\partial a_{e}}\right)>0 . \\
& \frac{\partial t_{f}^{*}}{\partial a_{e}}=\frac{b a_{e f}}{|H|}>0,
\end{aligned}
$$

As in the case of the two modes being substitutes, the following comparative static result is needed to determine the effect of advances in information technology on city size,

$$
\frac{\partial t_{e}^{*}}{\partial b}=\frac{c a_{e f}}{|H|}>0 .
$$

Result (15) implies that improvements in communications technology will increase the time spent on electronic communications since, as stated above, now it's easier and faster to communicate electronically. Therefore, if faceto-face interactions and electronic communications are complements, improvements in communications technology will increase the time spent on face-to-face interactions, which is what result (16) implies. Finally, result (17) implies that an increase in the productive time of face-to-face contact will increase the time spent on electronic contact.

From both general models we can conclude that improvements in communications technology unambiguously increases the time spent on electronic communications. However, improvements in communications technology increase the time spent on face-to-face interactions only if face-to-face interactions and electronic interactions are complements. If they are substitutes then improvements in communications technology results in reduction of time spent on face-to-face interactions.

\section{THE URBAN MODEL}

In this section we placed the above developed payoff model within a spatial framework in order to examine how changes in electronic communications affect the growth of cities.

Urban Model I (Face-to-face interactions and electronic communications are substitutes): The net payoff of relationships for the individual living in the city center is given by the following function,

$\Pi^{c}=a_{e} t_{e}^{c}+a_{f} b^{c} t_{f}^{c}-\frac{1}{2} a_{e e}\left(t_{e}^{c}\right)^{2}-\frac{1}{2} a_{f f}\left(b^{c} t_{f}^{c}\right)^{2}-a_{e f} t_{e}^{c} b^{c} t_{f}^{c}-c\left(t_{e}^{c}+t_{f}^{c}\right)-H N-R$,

while the net payoff of relationships for the individual living in the hinterland is given by the following function, 


$$
\Pi^{h}=a_{e} t_{e}^{h}+a_{f} b^{h} t_{f}^{h}-\frac{1}{2} a_{e e}\left(t_{e}^{h}\right)^{2}-\frac{1}{2} a_{f f}\left(b^{h} t_{f}^{h}\right)^{2}-a_{e f} t_{e}^{h} b^{h} t_{f}^{h}-c\left(t_{e}^{h}+t_{f}^{h}\right)-R,
$$

where $\mathrm{R}$ denotes the agricultural rent (i.e., is the housing cost beyond the edge of the city), $\mathrm{N}$ is the total population of the city and HN is commuting cost. We assume here that the city facilities face-to-face interactions and thus $b^{c}>b^{h}$, where $b^{c}$ is the fraction of face-to-face interactions that is productive in the city and $b^{h}$ is the fraction of face-to-face communications that is productive in the hinterland. Except for the cost differences and the productivity of face-to-face interactions, the two locations are identical.

The spatial equilibrium is found by equating the payoff in the two locations, $\Pi^{c}=\Pi^{h}$, i.e.,

$$
\begin{aligned}
& a_{e} t_{e}^{c}+a_{f} b^{c} t_{f}^{c}-\frac{1}{2} a_{e e}\left(t_{e}^{c}\right)^{2}-\frac{1}{2} a_{f f}\left(b^{c} t_{f}^{c}\right)^{2}-a_{e f} t_{e}^{c} b^{c} t_{f}^{c}-c\left(t_{e}^{c}+t_{f}^{c}\right)-H N-R= \\
& a_{e} t_{e}^{h}+a_{f} b^{h} t_{f}^{h}-\frac{1}{2} a_{e e}\left(t_{e}^{h}\right)^{2}-\frac{1}{2} a_{f f}\left(b^{h} t_{f}^{h}\right)^{2}-a_{e f} t_{e}^{h} b^{h} t_{f}^{h}-c\left(t_{e}^{h}+t_{f}^{h}\right)-R,
\end{aligned}
$$

which implies that

$$
N=\frac{1}{H}\left[\begin{array}{l}
a_{e} t_{e}^{c}+a_{f} b^{c} t_{f}^{c}-\frac{1}{2} a_{e e}\left(t_{e}^{c}\right)^{2}-\frac{1}{2} a_{f f}\left(b^{c} t_{f}^{c}\right)^{2}-a_{e f} t_{e}^{c} b^{c} t_{f}^{c}-c\left(t_{e}^{c}+t_{f}^{c}\right)- \\
a_{e} t_{e}^{h}-a_{f} b^{h} t_{f}^{h}+\frac{1}{2} a_{e e}\left(t_{e}^{h}\right)^{2}+\frac{1}{2} a_{f f}\left(b^{h} t_{f}^{h}\right)^{2}+a_{e f} t_{e}^{h} b^{h} t_{f}^{h}+c\left(t_{e}^{h}+t_{f}^{h}\right)
\end{array}\right]
$$

Differentiating (21) with respect to $a_{e}$ yields,

$$
\frac{\partial N}{\partial a_{e}}=\frac{1}{H}\left[\begin{array}{l}
\frac{\partial t_{e}^{c}}{\partial a_{e}}\left(a_{e}-a_{e e} t_{e}^{c}-a_{e f} b^{c} t_{f}^{c}-c\right)+\frac{\partial t_{f}^{c}}{\partial a_{e}}\left(a_{f} b^{c}-a_{f f}\left(b^{c}\right)^{2} t_{f}^{c}-a_{e f} b^{c} t_{e}^{c}-c\right)+t_{e}^{c}- \\
\frac{\partial t_{e}^{h}}{\partial a_{e}}\left(a_{e}-a_{e e} t_{e}^{h}-a_{e f} b^{h} t_{f}^{h}-c\right)-\frac{\partial t_{f}^{h}}{\partial a_{e}}\left(a_{f} b^{h}-a_{f f}\left(b^{h}\right)^{2} t_{f}^{h}-a_{e f} b^{h} t_{e}^{h}-c\right)-t_{e}^{h}
\end{array}\right]
$$

Noting from the FOCs that the terms in the parentheses are zero, the above expression (22) simplifies to

$$
\frac{\partial N}{\partial a_{e}}=\frac{1}{H}\left[t_{e}^{c}-t_{e}^{h}\right]
$$

The condition for equation (23) to be positive is that individuals in the city spend more time in electronic interactions. However, this result will not hold because from the general model I, if the two modes of interaction are substitutes then $\frac{\partial t_{e}}{\partial b}<0$, (result (10)), implying that the city residents spend less time in electronic interactions than the residents of the hinterland and that improvements in electronic communications cause the city to shrink.

Urban Model II (Face-to-face interactions and electronic communications are complements): In this case, the net payoff of relationships for the individual living in the city center is given by the following function, 
$\Pi^{c}=a_{e} t_{e}^{c}+a_{f} b^{c} t_{f}^{c}-\frac{1}{2} a_{e e}\left(t_{e}^{c}\right)^{2}-\frac{1}{2} a_{f f}\left(b^{c} t_{f}^{c}\right)^{2}+a_{e f} t_{e}^{c} b^{c} t_{f}^{c}-c\left(t_{e}^{c}+t_{f}^{c}\right)-H N-R$,

while the net payoff of relationships for the individual living in the hinterland is given by the following function,

$\Pi^{h}=a_{e} t_{e}^{h}+a_{f} b^{h} t_{f}^{h}-\frac{1}{2} a_{e e}\left(t_{e}^{h}\right)^{2}-\frac{1}{2} a_{f f}\left(b^{h} t_{f}^{h}\right)^{2}+a_{e f} t_{e}^{h} b^{h} t_{f}^{h}-c\left(t_{e}^{h}+t_{f}^{h}\right)-R$.

The spatial equilibrium is found by equating the payoff in the two locations, $\Pi^{c}=\Pi^{h}$, i.e.,

$$
\begin{aligned}
& a_{e} t_{e}^{c}+a_{f} b^{c} t_{f}^{c}-\frac{1}{2} a_{e e}\left(t_{e}^{c}\right)^{2}-\frac{1}{2} a_{f f}\left(b^{c} t_{f}^{c}\right)^{2}+a_{e f} t_{e}^{c} b^{c} t_{f}^{c}-c\left(t_{e}^{c}+t_{f}^{c}\right)-H N-R= \\
& a_{e} t_{e}^{h}+a_{f} b^{h} t_{f}^{h}-\frac{1}{2} a_{e e}\left(t_{e}^{h}\right)^{2}-\frac{1}{2} a_{f f}\left(b^{h} t_{f}^{h}\right)^{2}+a_{e f} t_{e}^{h} b^{h} t_{f}^{h}-c\left(t_{e}^{h}+t_{f}^{h}\right)-R,
\end{aligned}
$$

which implies that

$$
N=\frac{1}{H}\left[\begin{array}{l}
a_{e} t_{e}^{c}+a_{f} b^{c} t_{f}^{c}-\frac{1}{2} a_{e e}\left(t_{e}^{c}\right)^{2}-\frac{1}{2} a_{f f}\left(b^{c} t_{f}^{c}\right)^{2}+a_{e f} t_{e}^{c} b^{c} t_{f}^{c}-c\left(t_{e}^{c}+t_{f}^{c}\right)- \\
a_{e} t_{e}^{h}-a_{f} b^{h} t_{f}^{h}+\frac{1}{2} a_{e e}\left(t_{e}^{h}\right)^{2}+\frac{1}{2} a_{f f}\left(b^{h} t_{f}^{h}\right)^{2}-a_{e f} t_{e}^{h} b^{h} t_{f}^{h}+c\left(t_{e}^{h}+t_{f}^{h}\right)
\end{array}\right]
$$

Differentiating (27) with respect to $a_{e}$ yields,

$$
\frac{\partial N}{\partial a_{e}}=\frac{1}{H}\left[\begin{array}{l}
\frac{\partial t_{e}^{c}}{\partial a_{e}}\left(a_{e}-a_{e e} t_{e}^{c}+a_{e f} b^{c} t_{f}^{c}-c\right)+\frac{\partial t_{f}^{c}}{\partial a_{e}}\left(a_{f} b^{c}-a_{f f}\left(b^{c}\right)^{2} t_{f}^{c}+a_{e f} b^{c} t_{e}^{c}-c\right)+t_{e}^{c}- \\
\frac{\partial t_{e}^{h}}{\partial a_{e}}\left(a_{e}-a_{e e} t_{e}^{h}+a_{e f} b^{h} t_{f}^{h}-c\right)-\frac{\partial t_{f}^{h}}{\partial a_{e}}\left(a_{f} b^{h}-a_{f f}\left(b^{h}\right)^{2} t_{f}^{h}+a_{e f} b^{h} t_{e}^{h}-c\right)-t_{e}^{h}
\end{array}\right]
$$

Noting from the FOCs that the terms in the parentheses are zero, the above expression (28) simplifies to

$$
\frac{\partial N}{\partial a_{e}}=\frac{1}{H}\left[t_{e}^{c}-t_{e}^{h}\right]
$$

We can see that equations (23) and (29) are identical. This implies that the only requirement for cities to rise in population with telecommunications technology is just that individuals in the city spend more time in electronic interactions. In contrast to (23), this result in this case holds. As shown in the general model II, if the two modes of interaction are complements then $\frac{\partial t_{e}}{\partial b}>0$, (result (17)), implying that the city residents spend more time in electronic interactions than the residents of the hinterland and that improvements in electronic communications cause the city to grow.

From both urban models we can conclude that if face-to-face interactions and electronic communications are complements then improvements in telecommunications technology will increase city size, while if they are substitutes then improvements in telecommunications technology will decrease city size. 


\section{CONCLUSION}

In this paper we extended the Panayides (2002) and Gaspar and Glaeser (1998) models in examining the interdependence between face-to-face interactions and improvements in telecommunication technology. First, a general model was developed which showed that improvements in communications technology increases the time spent on electronic interactions, but the effect on face-to-face interactions is ambiguous. Specifically, improvements in communications technology increase the time spent on face-to-face interactions only if the two modes of interaction are complements. The general model was then placed within an urban framework to show that if the face-to-face interactions and electronic communications are complements then improvements in telecommunications technology increases city size.

\section{AUTHOR INFORMATION}

Dr. Alexandros Panayides earned his Ph.D. from Binghamton University (SUNY Binghamton) in 2000 and he is currently an Associate Professor and the Chairperson of the Department of Economics, Finance and Global Business at William Paterson University.

\section{REFERENCES}

1. Gaspar and Glaeser (1998), "Information Technology and the Future of Cities", Journal of Urban Economics, 43, 136-156.

2. Knoke, K. (1996), Bold New World: The Essential Road Map to the Twenty-First Century, Kodansha, New York.

3. Naisbitt, R. (1995), The Global Paradox, Avon Books, New York.

4. $\quad$ Negroponte, N. (1995), Being digital, Vintage Books, New York.

5. Panayides, A. (2002), "The Effects of Improvements in Information Technology on Growth of Cities: An Extension," International Business and Economics Research Journal, 11, 41-48

6. Toffler, A. (1980), The Third Wave, Bantam Books, New York. 


\section{NOTES}

